\title{
Searching for the Reionization Epoch
}

\author{
P. A. Shaver \\ European Southern Observatory, Karl-Schwarzschild-Str. 2, D-85748 \\ Garching bei München, Germany
}

\begin{abstract}
Recent advances have led to the discoveries of both galaxies and quasars out to $z \sim 6$. The reionization epoch itself, thought to lie somewhere in the range $6<z_{i}<15$, may be within reach over the next decade. Prospects for the detection of the first luminous objects in the Universe are reviewed - the objects that reionized the Universe and brought an end to the "Dark Ages". Alternative possibilities for detecting the reionization epoch are also discussed, including direct detection of the intergalactic medium itself at that epoch.
\end{abstract}

\section{Introduction}

The reionization epoch was one of the major phases in the history of the Universe. It marked the end of the "Dark Ages", during which the primordial background cooled and shifted into the infrared and radio. Darkness persisted until early structures collapsed and formed the first stars and quasars that reionized the Universe.

The redshift of reionization is broadly constrained observationally. Clearly it is above $z \sim 6$, from the observed spectra of known galaxies and quasars and the measured high degree of ionization of the intergalactic medium. On the other hand it cannot be much above $z \sim 30$, as otherwise Thomson scattering by the ionized intergalactic medium would have damped out the fluctuations observed in the microwave background.

Analytical and numerical estimates typically give $6<z_{i}<15$ (e.g., Gnedin \& Ostriker 1997; Haiman \& Loeb 1998; Valageas \& Silk 1999; Gnedin 2000). Gnedin (2000) describes in detail three stages in the reionization process: (i) pre-overlap $(z \sim 15->7$ ), which occurred in about the (then) Hubble time, (ii) overlap $(z \sim 7)$, when the $\mathrm{H}_{\text {II }}$ regions forming around the ionizing sources overlapped each other, and which occurred very suddenly (in about $10 \%$ of the Hubble time, almost like a "phase transition" of the Universe), and (iii) postoverlap $(z \sim 7->)$. The reionization redshift $z_{i}$ is defined to be that of the rapid overlap phase.

A brief review is given below of the progress leading to the highest-redshift objects known today, our knowledge of the evolution of these objects at high redshift, and the prospects for detecting both the first luminous sources and the intergalactic medium itself at the time of reionization. 


\section{The Advance to High Redshifts}

The high-redshift Universe has been opened up by a burst of discoveries and developments over the last decade. For many years the highest-redshift objects known were quasars found in radio surveys. Redshifts in the vicinity of 2 were found quickly after the discovery of quasars, indicating the rapid increase in space density that is well known today. From then on progress was slow. New optical search techniques dedicated to finding high-redshift quasars proved successful in the late 1980s, and resulted in the discovery of quasars with redshifts up to 5 . Recently the Sloan survey has found several quasars at $z>5$, including the highest-redshift known quasar at $z=5.8$ (Fan et al. 2000a), and other new deep surveys are also underway. However, it has taken 35 years of dedicated effort to go from $z \sim 2$ to $z \sim 6$, compared with less than two years to go from the original discovery of quasars at $z \sim 0.1$ to reach $z \sim 2$. This is a reflection of the fact that the rapid increase in space density of quasars out to $z=2$ flattens off and then probably declines at higher redshifts.

Galaxies have been far more difficult to detect at high redshifts. Through the 1970s and 1980s the highest-redshift galaxies known were again found by virtue of their radio emission. Luminous radio galaxies were detected and studied in detail out to redshifts of $1-2$. When it was realised that the highest-redshift radio galaxies have very steep radio spectra this came to be used as a search criterion, with great success, and objects at $z \sim 4-5$ were found. However, the major breakthrough came with the realization that high-redshift galaxies could be found in vast numbers using the Lyman break as a signature, and using the powerful new telescopes that became available in the 1990s, particularly the HST and Keck. There are now hundreds of galaxies known at $z>4$, and even several at $z>5$. These include a radiogalaxy at $z=5.19$ (van Breugel et al. 1999a, b), V-band dropouts at $z=5.34$ (Spinrad et al. 1999), a multicolour-selected galaxy at $z=5.6$ (Weymann et al. 1998), a Ly $\alpha$-selected galaxy at $z=5.74(\mathrm{Hu}$ et al. 1999b), and possibly an I-band dropout at $z=6.68$ (Chen et al. 1999).

\subsection{Evolution at High Redshift}

It is important to know the evolution of such objects at high redshifts, as this gives us an understanding of the processes involved in the early formation of stars, galaxies and quasars. Above some redshift the space density of these luminous objects should decrease, and this will give clues to the epoch of reionization. In the case of luminous quasars, a steep decline above $z \sim 3$ has long been indicated by optically-selected samples (Warren et al. 1995; Schmidt et al. 1995; Kennefick et al. 1995). A similar result is found from radio samples, indicating that the decline is real, and not merely due to obscuration by intervening dust (Dunlop \& Peacock 1990; Shaver et al. 1996).

Recent results from the Sloan survey have dramatically confirmed these earlier conclusions. The same survey that has been so successful in finding the highest-redshift quasars has also now confirmed that the number of luminous quasars drops off steeply at high redshift (Fan et al. 2000b). The "rise and fall" of quasars - the quasar epoch - seems now to be well established, at least for the most luminous quasars. 
X-ray surveys provide another powerful way to find quasars, as virtually all quasars are X-ray sources, and such surveys are now sufficiently sensitive to reach to high redshifts. A recent analysis of the ROSAT samples did not find a significant drop in the space density of X-ray quasars at high redshift (Miyaji et al. 1999), although the numbers were small and the result only marginally inconsistent with the optical and radio samples. Hence the difference could be merely statistical, but Miyaji et al. suggest that it could also be due to the lower intrinsic luminosity of the X-ray quasars and a change in the slope of the quasar luminosity function at high redshift - the turnover in space density may be not as pronounced for objects of lower luminosity. The newer X-ray satellites (Chandra and XMM) have the sensitivity to reach out to the highest redshifts, and should soon resolve this issue.

The large samples of high-redshift galaxies becoming available have been used to estimate the evolution of the global star formation rate, which was initially found to peak at $z \sim 1-2$ and fall off at higher redshifts, strikingly similar to the space distribution of quasars (Madau et al. 1996). However, the observations of these galaxies were all in the rest-frame UV and therefore highly susceptible to reddening by dust in the galaxies; adjustments had to be made to allow for this absorption. Furthermore, recent studies of brighter galaxies (Steidel et al. 1999) have shown no significant decline in the star formation rate at $z>4$. The difference between these results could be due to luminosity dependence, or possibly sample variance in the small HDF field used for the original Madau et al. study. Preliminary results from still more recent work by $\mathrm{Hu}$ et al. (1999a) indicates that the space density of Ly $\alpha$-emitting galaxies may decrease by a factor of six from $z \sim 4$ to $z \sim 6$, while photometric studies by Lanzetta et al. $(1998,1999)$ suggest a continuing increase in the luminosity density to beyond $z=5$, and an upper limit on galaxies at very high redshift.

A new window on star formation at high redshifts is provided by observations in the $\mathrm{mm} / \mathrm{submm}$ wavelength range with bolometer arrays such as SCUBA on the JCMT and MAMBO on the IRAM 30-m telescope. These observations benefit from the negative K-correction for high-redshift star-forming galaxies caused by the large far infrared dust-emission peak being redshifted into the $\mathrm{mm} / \mathrm{submm}$ bands: galaxies at high redshift can actually appear brighter than they would at low redshift. So far it has been difficult to draw concrete conclusions from these observations because of the difficulty of making optical identifications and redshift determinations. Nevertheless these observations do suggest the presence of a large (possibly dominant) population of dusty starforming galaxies at $z \sim 3$ and perhaps higher.

Thus, while the decline in space density of luminous quasars at high redshifts appears to be confirmed, current evidence on the evolution of less luminous quasars, galaxies, and the global star formation rate is mixed, and the complete picture on evolution at high redshift remains to be determined.

\section{Detecting the Reionization Epoch}

Fossil evidence for the presence of luminous sources at $z>6$ abounds. Heavy elements are found in the emission spectra of high redshift quasars and widely distributed in quasar absorption systems up to the highest accessible redshifts, 
indicating nucleosynthesis in stars at still earlier epochs. Observations also indicate that the intergalactic medium was already highly ionized by $z \sim 5$ (e.g., Songaila et al. 2000). However, studies of the overall blanketing of quasar and galaxy spectra due to the intervening Ly $\alpha$ forest clouds (e.g., Spinrad et al. 1999, Fig. 4) show a rapid increase up to the highest accessible redshifts $(>80-90 \%$ at $z \sim 5-6$ ), suggesting that the reionization epoch may not be far beyond.

Indeed, the He II reionization epoch may already have been found. The observed pronounced differences between the H I Ly $\alpha$ forest and the He II $304 \AA$ forest found by Reimers et al. (1997) in a quasar spectrum at $z \sim 3$ suggest that He II was only partially ionized at that redshift. Heap et al. (2000) find that the He II Ly $\alpha$ opacity drops significantly below $\mathrm{z}=3$, while the hardness of the UV background may increase rapidly according to Songaila's (1998) measurements of the S IV/C IV ratio. Finally, from studies of the widths of Ly $\alpha$ forest lines Schaye et al. (2000) claim that there is a peak in the temperature of the intergalactic medium at $z \sim 3$, which they attribute to reheating by the reionization of He II. A helium reionization epoch at $z \sim 3$ would be consistent with the peak in the quasar space density at a similar redshift.

There are two broad possibilities for detecting the reionization epoch: searching for the discrete sources that formed at that time and caused the reionization, and searching for large - in some cases global - features from the intergalactic medium itself. These are discussed in the two sections below.

\subsection{Discrete Sources}

What types of sources are likely to have dominated the reionization process? Only trace amounts of bayrons are required to ionize the Universe, as nuclear fusion and black-hole accretion release $>10^{6} \mathrm{eV}$ per hydrogen atom, compared with $13.6 \mathrm{eV}$ for ionization. According to the conventional scenario, small $\left(T_{v i r}<10^{4} \mathrm{~K}\right)$ halos first collapsed by $\mathrm{H}_{2}$ cooling. This process was then suppressed by photodissociation of $\mathrm{H}_{2}$ due to photons of $11.2-13.6 \mathrm{eV}$ produced by the first sources (Haiman, Abel, \& Rees 2000$)$. Later, large $\left(T_{v i r}>10^{4} \mathrm{~K}\right)$ halos were able to collapse by atomic cooling. The first ionizing sources may well have been stars rather than quasars.

There is some observational evidence suggesting that quasars did not dominate in the reionization epoch (e.g., Madau, Haardt, \& Rees 1999). Limits on the number of faint stellar objects in the Hubble Deep Field are inconsistent with quasars having caused the reionization of the Universe (Haiman, Madau, \& Loeb 1999). The X-ray background also imposes a limit on the possible density of quasars at high redshifts (Haiman \& Loeb 1999b). The evidence reported above in favour of He II reionization at $z \sim 3$ indicates that the helium and hydrogen reionization epochs were probably different, in contrast to what would be expected if quasars had dominated the reionization process. And the presence of metals in widely distributed low column density absorption systems, as reported by Cowie in these proceedings, seems more consistent with a large, widespread population of early stars than a more sparse population of luminous AGN. The present evidence thus suggests that stars may have dominated the reionization process.

Nevertheless, while very luminous quasars may not have been commonplace in the reionization epoch, there could still have been a substantial popula- 
tion of low-luminosity "mini"-quasars at high redshifts. The presently ongoing searches based on optical/near-infrared and radio surveys may succeed in finding further rare quasars at higher redshifts. The Sloan survey is currently very productive, and other dedicated searches continue. The vast and sensitive radio surveys that now cover the sky provide a huge source list for increasingly efficient optical/near-infrared (and eventually $\mathrm{mm} / \mathrm{submm}$ ) follow-up observations. These radio surveys are unaffected by dust obscuration, and are sensitive to AGN out to the highest redshifts. X-ray searches for high-redshift quasars are now coming of age, with the sensitivity, angular resolution and positional accuracy to discover AGN out to very high redshift. Haiman \& Loeb (1999b) predict that the Chandra satellite may detect as many as 100 quasars at $z>5$ per $17^{\prime} \times 17^{\prime}$ field of view. Some have probably already been detected, and just await spectroscopic confirmation. A decade from now NGST may also detect $\sim 10^{2}$ (mini)quasars at $z>5$ per $5^{\prime} \times 5^{\prime}$ FOV (Haiman \& Loeb, 1999c), and the Atacama Large Millimeter Array (ALMA) should detect the heated dust associated with star formation in these objects.

The spectroscopic identification of (mini)quasars near the reionization redshift may pose a significant challenge. The Ly $\alpha$ emission line may be obscured by the strong damping wing of the Gunn-Peterson trough (Haiman \& Loeb 1998, 1999a). The highest-redshift sources may appear as little more than continuum steps, with no Ly $\alpha$ line and no emission to the blue. In the case of a sufficiently luminous quasar, however, the ionized zone produced may be large enough that the G-P damping wing does not reach to the redshift of the quasar, so that a Ly $\alpha$ emission line would still be observable (Madau \& Rees 2000; Cen \& Haiman 2000).

Galaxies have been detected up to $z=5.74$, and galaxies at still higher redshifts may be detectable with current techniques, although this is becoming difficult even with 8-10 m class telescopes. Broadband techniques give possible photometric redshifts at $z>6$ (Yahata et al. 2000), although these remain to be confirmed.

Another promising possibility is narrow-band imaging, with the band placed between the night sky lines ( $\mathrm{Hu}$ et al. 2000), but this requires a strong and unobscured Ly $\alpha$ emission line. In the future, NGST will make it possible to detect the sub-components of forming galaxies at high redshift: Haiman \& Loeb (1999c) have estimated that as many as $10^{3}-10^{4}$ mini-galaxies may be detectable at $z>5$ per FOV.

A possible problem for these wavebands is that dust created by the first stars could produce some opacity even in the near-infrared, which corresponds to the rest-frame ultraviolet at $z \sim 10$. In the $\mathrm{mm} / \mathrm{submm}$ wavebands, the same dust that obscures the optical/near-infrared in star-forming galaxies produces the redshifted far-infrared peak that makes it possible to detect very distant objects. ALMA will have the sensitivity and angular resolution to detect the dust emission from early protogalaxies, and the "ladder" of molecular transitions will make it possible to measure redshifts from the (sub) $\mathrm{mm}$ observations alone.

Mapping the redshift evolution of the global star formation rate may provide a signature of reionization (Barkana \& Loeb 2000). A dip in the global SFR may be present at reionization due to an increased $T_{I G M}$ suppressing galaxy formation. This dip would give the reionization redshift. 
Individual clusters of metal-free massive stars at $z \sim 10$ may be detectable with NGST (Bromm et al. 2000). With $T_{\text {eff }} \sim 10^{5} \mathrm{~K}$ they would produce strong hydrogen and helium recombination lines in the surrounding ISM. However, as in the case of quasars, it is unlikely that such objects could have dominated the overall reionization process, as they would have reionized hydrogen and helium together, whereas the observations appear to favour delayed reionization of helium.

Supernovae and gamma-ray bursts should be detectable at high redshifts. NGST may be able to detect tens of high-redshift supernovae per year in its $5^{\prime} \times 5^{\prime}$ field of view (Rees 1998). If gamma-ray bursts are associated with massive stars, they may also have occurred in the early star-formation epoch. Their afterglows may be readily detectable (Lamb \& Reichart 2000a, b; Ciardi \& Loeb 2000). Although rare and transitory, even one such object at the reionization epoch could provide a wealth of information about the intervening medium through absorption-line spectroscopy.

Both emission lines and continuum radiation may be detectable from the ionized halos surrounding the first luminous sources. $\mathrm{H} \alpha$ and $\mathrm{H} \beta$ may be detectable by NGST (Oh 1999), and they would be easier to interpret than Ly $\alpha$ which is subject to obscuration by dust and the damping wing of the GunnPeterson trough. He II recombination lines may also be detectable from sources of hard radiation, such as metal-free starbursts (Oh et al. 2000). Free-free continuum emission at radio wavelengths may be detectable from $\sim 10^{4}$ sources at $z>5$ above $70 \mathrm{nJy}$ in the $1 \mathrm{deg}^{2}$ field of the Square Kilometer Array (SKA) (Oh 1999). Radio recombination line maser emission might be produced by early H II regions of high emission measure. The required anisotropy may be present at the $\mathrm{H}_{\mathrm{II}}-\mathrm{H}_{\mathrm{I}}$ interfaces, where the pump photons can escape. Spaans \& Norman (1997) have estimated that $n \sim 100$ states might produce lines at $50-200 \mathrm{MHz}$ that would be detectable with SKA. And scattering of Ly $\alpha$ photons from sources before reionization may produce halos around those sources (Loeb \& Rybicki 1999). Diffuse $\left(\sim 15^{\prime \prime}\right)$ Ly $\alpha$ halos may be detectable from very luminous sources, and would be highly polarized, up to $60 \%$ (Rybicki \& Loeb 1999). Given their expected faintness, however, it will probably be more productive to look for such halos when the sources themselves are known, rather than searching for the halos first.

Finally, $\mathrm{HI}$ emission and absorption shells may be detectable around early quasars (Madau et al. 1997; Tozzi et al. 2000). As discussed below, the H I spin temperature near reionization can be coupled to the kinetic temperature of the gas through scattering by Ly $\alpha$ photons. In the vicinity of a quasar, the gas is heated by soft X-rays from the quasar itself. Further away, the temperature of the IGM may be lower than that of the CMB. Thus, the quasar will be surrounded by a zone of $\mathrm{HI}$ emission, and this in turn could be surrounded by a ring of $\mathrm{HI}$ absorption, since the Ly $\alpha$ photons can reach regions where $T_{K}<T_{C M B}$. Simulations of these effects have been made by Tozzi et al. (2000) and Meiksin (1999); they may be detectable with the Square Kilometer Array. 


\subsection{Large-Scale Features}

A different approach to detecting the epoch of first light is to look for largescale features caused directly or indirectly by the reionization process. Various possibilities that have been proposed are discussed below.

The Global HI Signal A global spectral feature from the reionization epoch may be present over the whole sky. If reionization took place at $5<z_{i}<20$, a step should be present in the radio spectrum of the sky at $70<\nu<240 \mathrm{MHz}$ due to redshifted H I 21-cm line emission. The expected amplitude is well above fundamental detection limits, and the sharpness of the feature may make it distinguishable from variations due to galactic and extragalactic foregrounds (Shaver et al. 1999).

In the absence of a decoupling mechanism, the spin temperature of neutral hydrogen would go to equilibrium with the CMB, and no emission or absorption relative to the CMB would be detected. Scattering by Ly $\alpha$ photons provided a mechanism to couple the spin temperature to the kinetic temperature of the gas, which could have been above or below the CMB temperature (Madau et al. 1997; Tozzi et al. 2000). Thus, the neutral IGM just prior to reionization may have produced a copious 21-cm line signal from neutral hydrogen (in emission or absorption), a signal which would have largely disappeared following reionization. A signature ("step") in the continuum spectrum of the radio sky at $\sim 70-240 \mathrm{MHz}$ will therefore flag the reionization epoch.

The sharpness of this step depends on several factors, including the clumpiness of the Universe at that time and the nature of the first luminous objects. If reionization was initiated by a population of sparsely distributed quasars, the "step" would be irregular over the sky, appearing at different redshifts in different directions. But if it was caused by a vast number of stars well-distributed spatially, it may have been well synchronized over the sky, producing a welldefined all-sky spectral feature.

The observed differential antenna temperature across the "step" is expected to be $\Delta T \sim 0.02 \mathrm{~K}$ (for $h=0.5$ ) at 70-240 MHz. The fundamental limitation on the sensitivity achievable is set by the intensity of the galactic and extragalactic foregrounds, which dominate the system temperature. The limiting sensitivity is $\Delta T_{\min } \sim T_{\text {sys }} / \sqrt{\delta \nu t}$, where $T_{\text {sys }}$ is the total system temperature, $\delta \nu$ is the bandwidth, and $t$ is the integration time. For $T_{\text {sys }}=150 \mathrm{~K}$ (the coldest regions of the sky at $150 \mathrm{MHz}$ ) with a bandwidth of $5 \mathrm{MHz}$ and integration time 24 hours, $\Delta T_{\min } \sim 0.0002 \mathrm{~K}$. The reionization step would therefore be $\sim 100 \sigma$, independent of telescope size. Clearly sensitivity is not an issue, and the challenges are signal contamination, calibration and interference.

The difficulty posed by the galactic and extragalactic foregrounds is that they can be complex, both in frequency and position. The question is whether a $0.02 \mathrm{~K}$ step can be extracted from this much stronger varying continuum. This emission is comprised of four components: galactic synchrotron emission ( $70 \%$ at $150 \mathrm{MHz}$ ), galactic thermal (free-free) emission $(\sim 1 \%)$, the integrated emission from extragalactic sources $(\sim 27 \%)$, and the cosmic background itself. Simulations that include all of these contaminating effects indicate that the $\mathrm{H} \mathrm{I}$ step from the reionization epoch may still be detectable because of its relative sharpness. The fact that it should be the same over the whole sky is of course 
a great advantage, as searches in different directions should give the same result. The remaining limitations are purely technical, and present an interesting challenge.

The main technical issues are calibration and interference. Calibrating the frequency response has to be done to better than a few parts in $10^{5}$ over a wide frequency range. Possibilities include internal loads, or the use of astronomical sources - a very strong 'featureless' continuum source such as Cas A. The Moon provides an interesting alternative. As it moves, a true differencing experiment could be done at exactly the same position in the sky. All stable contaminating effects (galaxy, discrete sources, sidelobes) should be virtually identical and cancel. Furthermore, detection of the signal could in principle be done interferometrically. Because the Moon occults the background signal it introduces spatial structure in the global edge signal. The spectrum of this interferometer signal contains the reionization step. Thus, use of the Moon can convert an inherently single-dish experiment into an interferometer experiment, and this has significant advantages.

Radio-frequency interference (RFI) is of course a major issue for observations at the frequencies of interest. In particular, the FM bands around $100 \mathrm{MHz}$ may be completely inaccessible for radio observations of the required accuracy (it would be most unfortunate if the reionization signal is in the corresponding redshift range of $\sim 12-15$ !). The use of interferometers can greatly help in coping with interference, and techniques are being developed to excise RFI from astronomical observations using variability, delay and fringe rate information.

Thus, while challenging, detection of the $\mathrm{HI}$ step from the reionization epoch might be possible with techniques that are currently being developed.

Fluctuations in the HI Emission Fluctuations should be present in this global $\mathrm{H}$ I signal, because of density inhomogeneities in the intergalactic medium. These fluctuations will be present both in frequency and in position across the sky. They will be very weak, but much stronger than the intrinsic CMB fluctuations. They correspond to linear scales of a few comoving $\mathrm{Mpc}$, and are in the linear regime at $z>5$. Thus, they will give a direct measure of the matter density field at that epoch, i.e., between the epoch probed by galaxy surveys and recombination. The expected brightness temperature fluctuations have been computed for a range of cosmological models by Tozzi et al. (2000) and Meiksin (1999).

Because of the small angular scale of these fluctuations (several arcmin) as well as their weakness (several milli-K), detection of these fluctuations will require very large radiotelescopes, in contrast to the global signal described above. The Square Kilometer Array should be capable of detecting the fluctuations in integration times of tens to hundreds of hours (e.g., Shaver \& de Bruyn 2000; Meiksin 1999). A more immediate prospect (cf. Briggs \& de Bruyn 2000) may be provided by the Low Frequency Array, LOFAR, an SKA precursor which will also have a very large collecting area at low frequencies and may be operational by 2006 . One would search for the fluctuations as features in three-dimensional (spatial and spectral) "data cubes" measured in the $70-240 \mathrm{MHz}$ range. Foreground contamination will also be an issue in this experiment. There will almost certainly be spatial fluctuations in the much stronger galactic emission at these frequencies, and if, as is likely, these are accompanied by spectral index 
variations, there will be associated fluctuations in the spectral domain as well, although presumably not as sharp as those due to the $\mathrm{H}$.

Global Lyman and Balmer Spectral Features There should also be global Lyman and Balmer spectral features present over the sky in the optical/nearinfrared. The rate of hydrogen recombinations is expected to have peaked at reionization. The optical depth in the Lyman series would have been very high at $z>z_{i}$; all Lyman series lines except $\operatorname{Ly} \alpha$ would have been absorbed immediately and redistributed. This would have made Ly $\alpha$ and the Balmer series significantly brighter, because they would have received the energy of the Lyman series. Once reionization occurred, however, the Lyman series would have become optically thin and the brightness of $\operatorname{Ly} \alpha$ and the Balmer series would have dropped considerably, producing sharp features in the background (Baltz et al. 1999). Broader features due to blends of lines from the Lyman and Balmer series should also be present; their shapes are determined by the evolution of the reionization/recombination history. A characteristic spectral pattern should therefore be present over the whole sky at optical/near-infrared wavelengths.

These features are expected to be very faint. Baltz et al. (1999) estimate $J_{\nu} \sim 0.3-3 \times 10^{-23} \mathrm{erg} / \mathrm{cm}^{2} / \mathrm{s} / \mathrm{Hz} / \mathrm{sr}$. Possibilities for detection have been considered by Shaver et al. (1999). An optical/near-infrared instrument in space is essential because of the low sky background and absence of terrestrial $\mathrm{OH}$ lines. The fundamental sensitivity limits for such an observation are then set by foreground emissions from the zodiacal light, the diffuse galactic light, and the extragalactic background light. A pilot experiment using 28 orbits in parallel mode with the Space Telescope Imaging Spectrograph (STIS) on HST has already been carried out (Windhorst et al. 2000). A $2 \sigma$ upper limit of $J_{\nu}=2 \times 10^{-21} \mathrm{erg} / \mathrm{cm}^{2} / \mathrm{s} / \mathrm{Hz} / \mathrm{sr}$ has been achieved, and observations reaching several times deeper are planned. Presumably fluctuations would also be expected in this optical/near-infrared signal, and these may make it easier to distinguish from the contaminating foreground emissions. Ultimately such experiments may require a "deep-space" infrared mission at several AU from the Sun, to sufficiently reduce the zodiacal foreground.

Effects on the Microwave Background Finally, the reionization epoch will leave its imprint on the cosmic microwave background. Thomson scattering from the free electrons will result in damping, Doppler and polarization effects (e.g., Haiman \& Knox 2000). This provides an additional probe of the reionization epoch, although it also contaminates the primary anisotropies from the CMB. Consideration of the damping that can be produced on the original CMB anisotropies has already provided important information about the reionization epoch by giving an upper limit to the redshift of reionization, $z_{i}<30$. The peak in the polarization power spectrum, if it could be measured, would also provide a determination of the epoch of reionization. Secondary anisotropies can be imprinted on the CMB as a result of the spatially varying ionization fraction, fluctuations which arise through the density and velocity variations associated with inhomogeneous reionization. These may be detectable with the Atacama Large Millimeter Array (Benson et al. 2000). 


\section{Summary}

Searches for distant galaxies and quasars are approaching the epoch of reionization. Observations of these very early objects are increasingly difficult even with the most powerful telescopes available today. However, the next generation of large ground- and space-based astronomical facilities should be capable of detecting the first luminous objects that reionized the Universe and brought an end to the "Dark Ages". It may also be possible to detect and study the intergalactic medium of that time through a variety of novel observations.

\section{References}

Baltz, E. A., Gnedin, N. Y., \& Silk, J. 1999, ApJ, 493, L1

Barkana, R., \& Loeb A. 2000, ApJ, 539, 20

Benson, A. J., Nusser, A., Sugiyama, N., \& Lacey, C. G. 2000, MNRAS, in press, astro-ph/0002457

Briggs, F., \& de Bruyn, A. G. 2000, in preparation

Bromm, V., Kudritzki, R. P., \& Loeb, A. 2000, ApJ, in press, astro-ph/0007248

Cen, R., \& Haiman, Z. 2000, ApJ, in press, astro-ph/0006376

Chen, H-W., Lanzetta, K.M., \& Pascarelle, S. 1999, Nature, 398, 586

Ciardi, B., \& Loeb, A. 2000, ApJ, 540, 687

Dunlop, J. S., \& Peacock J. A. 1990, MNRAS, 273, L63

Fan, X. et al. 2000a, AJ, in press, astro-ph/0005414

Fan X. et al. 2000b, AJ, in press, astro-ph/0008123

Gnedin, N. Y. 2000, ApJ, 535, 530

Gnedin, N. Y., \& Ostriker, J. P. 1997, ApJ, 486, 581

Haiman, Z., Abel T., \& Rees, M. J. 2000, ApJ, 534, 11

Haiman, Z., \& Knox L. 2000, in Microwave Backgrounds, ed. Oliviera-Costa \& Tegmark (San Francisco: ASP), in press, astro-ph/9902311

Haiman, Z., \& Loeb, A. 1998, ApJ, 503, 505

Haiman, Z., \& Loeb, A. 1999a, ApJ, 519, 479

Haiman, Z., \& Loeb, A. 1999b, ApJ, 521, L9

Haiman, Z., \& Loeb, A. 1999c, in AIP Conf. Proc., 470, After the Dark Ages, ed. S. Holt \& E. Smith (New York: AIP), 34

Haiman, Z., Madau, P., \& Loeb A. 1999, ApJ, 514, 535

Heap, S. R., Williger, G. M., Smette, A., Hubeny, I., et al. 2000, ApJ, 534, 69

Hu, E. M., Cowie, L. L., \& McMahon, R. G. 1999a, in ASP Conf. Ser. Vol. 193, The Hy-Redshift Universe, ed. A. J. Bunker \& W. J. M. van Breugel (San Francisco: ASP), 554

Hu, E. M., McMahon, R. G., \& Cowie, L. L. 1999b, ApJ, 522, L9

Kennefick, J. D., Djorgovski, S. G., \& de Carvalho, R. R. 1995, AJ, 110, 2553

Lamb, D.Q., \& Reichart, D. E. 2000a, in Proc. 10th Maryland Conf., Cosmic Explosions, in press, astro-ph/0002034 
Lamb, D.Q., \& Reichart, D. E. 2000b, in 5th Huntsville Gamma-Ray Burst Symposium, in press, astro-ph/0002035

Lanzetta, K. M., Yahil, A., \& Fernandez-Soto, A. 1998, AJ, 116, 1066, astro-ph 9806226

Lanzetta, K. M., et al. 1999, in ASP Conf. Ser. Vol. 193, The Hy-Redshift Universe, ed. A. J. Bunker \& W. J. M. van Breugel (San Francisco: ASP), 544

Loeb, A., \& Rybicki, G. B. 1999, ApJ, 524, 527

Madau, P., et al. 1996, MNRAS, 283, 1388

Madau, P., Haardt, F., \& Rees, M. J. 1999, ApJ, 514, 648

Madau, P., Meiksin, A., \& Rees, M. J. 1997, ApJ, 475, 429

Madau,, P., \& Rees, M. J. 2000, ApJ, in press, astro-ph/0006271

Meiksin, A. 1999, Invited review for the Square Kilometre Array Radio Telescope Science Case, ed. R. Braun \& A. R. Taylor, astro-ph/9902384

Miyaji, T., Hasinger, G., \& Schmidt, M. 1999, in Highlights of X-Ray Astronomy, ed. B. Aschenbach \& M. Freyberg (MPE Report 272), 222, astro$\mathrm{ph} / 9809398$

Oh, S. P. 1999 , ApJ, 527, 16

Oh, S. P., Haiman, Z., \& Rees, M. J. 2000, ApJ, in press, astro-ph/0007351

Rees, M. J. 1998, in Science with NGST (34th Liege Astr. Coll.; ESA SP-429), 5 , astro-ph/9809029

Reimers, D., et al. 1997, A\&A, 327, 890

Rybicki, G. B., \& Loeb, A. 1999, ApJ, 520, L79

Schaye, J., Theuns, T., Rauch, M., Efstathiou, G., \& Sargent, W. L. W. 2000, MNRAS, in press, astro-ph/9912432

Schmidt, M., Schneider, D. P., \& Gunn, J. E. 1995, AJ, 110, 68

Shaver, P. A., \& de Bruyn, A. G. 2000, in Perspectives on Radio Astronomy: Science with Large Antenna Arrays, ed. M. P. van Haarlem, ASTRON, 45

Shaver, P. A., Wall, J. V., Kellermann, K. I., Jackson C. A., \& Hawkins, M. R. S. 1996, Nature, 384, 439

Shaver, P. A., Windhorst, R. A., Madau, P., \& de Bruyn, A. G. 1999, A\&A, 345,390

Songaila, A. 1998, AJ, 115, 2184

Songaila, A., Hu, E. M., Cowie, L. L., \& McMahon, R. G. 2000, ApJ, 525, L5

Spaans, M., \& Norman, C. A. 1997, ApJ, 488, 27

Spinrad, H., et al. 1999, AJ, 116, 2617

Steidel, C. C., et al. 1999, ApJ, 519, 1

Tozzi, P., Madau, P., Meiksin, A., \& Rees, M. 2000, ApJ, 528, 597

Valageas, P., \& Silk, J. 1999, A\&A, 347, 1

van Breugel, W., et al. 1999a, in The Most Distant Radio Galaxies, ed. Rottgering, Best, \& Lehnert (Amsterdam: KNAW), 49 
van Breugel, W., et al. 1999b, in ASP Conf. Ser. Vol. 193, The Hy-Redshift Universe, ed. A. J. Bunker \& W. J. M. van Breugel (San Francisco: ASP), 44

Warren, S. J., Hewett, P. C., \& Osmer, P. S. 1995, ApJ, 421, 412

Weymann, R. J., et al. 1998, ApJ, 505, L95

Windhorst, R., Bernstein, R., Plait, P., Collins, N., et al. 2000, in preparation

Yahata, N., Lanzetta, K. M., Chen, H.-W., Fernandez-Soto, A., et al. 2000, ApJ, 538,493

\section{Discussion}

Leonid Ozernoy: It was shown in the middle of the 1970s that the intergalactic medium could be reionized by photons from first generation quasars and AGN or by intense star formation in young galaxies. Based on the currently known luminosity functions of galaxies and QSS, which of these sources would you consider to be the major contributor to re-ionization?

Peter Shaver: The evidence at present seems to favour reionization dominated by stars rather than AGN. Relevant references are given in the text (section 3.1).

Charley Lineweaver: You showed a plot from Spinrad et al. (1999) which exhibits the continuum decrement as a function of redshift due to the Lyman $-\alpha$ forest. You said that you doubt this can be extrapolated or used to trace the reionization. Why?

Shaver: I meant that it could probably not be extrapolated to identify the actual redshift of reionization.

Piero Madau: It is difficult to use the observed $D_{A}$ decrement as a probe of the reionizaton epoch. Since resonant scattering has such a large cross section, even a gas that is partially ionized (e.g., with ionization fraction 0.99 ) will cause significant absorption, and, if diffuse, a large Gunn-Peterson optical depth. 